\title{
RÉFLEXIONS SUR L'ACTE COMPOSITIONNEL
}

Mik'hail Malt

(IRCAM)

RÉSUMÉ: Ce texte prétend montrer que l'idée de modèle et de formalisation font partie de ce que nous appelons le métier de compositeur et présente une vision de l'acte compositionnel que, nous espérons, pourra nous aider à mieux comprendre la place de l'ordinateur dans cette nouvelle discipline qu'est la Composition Assistée par Ordinateur.

\section{I.Les Jeux phases}

Notre lyypothèse de base est la possibilité de "décomposer" l'acte compositionnel en deux phases principales, pas nécessairement consécutives, ni disjointes, mais étroitement reliées entre elles:

La plase conceptuelle et La phase d'écriture.

\subsection{Les concepts}

La phase conceptuelle, est le moment où sont élaborées les grandes lignes de la composition, où sont mis en jeu les concepts, les idées de fond et où est èlaborée une espèce de thíorie, théorie prescriptive selon Déliège,' qui conduira à l'oeuvre.

Une Théorie prescriptive 2 est un ensemble de principes qui donnent des directives a priori, qui voudraient (dans le cas musical) construire un langage. Dans l'histoire de la musique nous avons eu un grand nombre d'exemples de théories de ce genre développées par des compositeurs, soit pour résoudre des problèmes spécifiques, suit pour proposer des nouveaux langages. Par exemple, la théorie nthmique du xiv siecle développée par Philippe de Vitry, le dodécaphonisme et le sćrialisme développés successivement par Schoenberg, Messiaen, Boulez et Stockhausen, la musique concrète de Schaeffer, le méconnu "System of Musica! composition" dév'eloppé par Joseph Schillinger entre les années 1920 et 1930 aux USA," et la musique stochastique préconisée par Xenakis. La prenière moitié du xx siècle a été sans doute le moment où les compositeurs se sont comportés le plus, comme des créateurs de langages, et où l'axiomatique a, en quelques moments, conquis des droits semblables a ceux de la perception.

Les réflexions de Schoenberg vis-à-vis de la hiérarchic tonale, celles de XENAKIS à l'égard de l'juée de "nuage", en sont des exemples de cette premiere phase, où la ríalisation pratique n"est pas en jeu, ce qui importe est de degager un ensemble de relations, de notions qui seront, en qutelque sorte, le support intellectuel de l'oeuvre.

\subsection{L'écriture}

$\dot{A}$ lopposé nous avons la planse "d "ecriture", moment où les concepts prendront forme (ou peut être, corps?) pour constituer l'oeuvre. Cette phase sera celle de la matérialisation des idées!

\footnotetext{
1 Célestin Déliege, musicologue belge.

2 DELIEGE Cílestin, (1991); Les thiories er leur projet Epistimologigne, Sśminaire de Doctorat pour le doctoral de Musigue tu Musicologie du xx siecle, le 07 novembre 1991, IRCAN1, l'atis.

3 SCIIILLINGER Josepli (1978). The Schillinger Sistem of M/tisical comprosition, Da Capo, New York.
} 
Il est important de souligner que nous pouvons utiliser le mot écriture avec deux sens: Écriture au sens graphique, c.-̀̀-d., comme moyen de notation, et écriture au sens compositionnel, comme étant une procédure à travers laquelle le compositeur réussi à représenter ses concepts sur un support quelconque.

Dans notre cas précis, c'est la deuxième signification que nous allons utiliser pour le mot "écriture", en admettant que l'écriture, au sens graphique, n'est qu'une des manières possibles, à un compositeur, pour exprimer sa pensée. Le montage sonore analogique sur une bande, le mixage numérique dans des logiciels tels que Pro-Tools," et l'ćcriture, au sens usuel du terme, sont des scénarios possibles pour ce que nous avons appelé la phase d'écriture.

La phase d'écriture, sera la phase de réalisation. Nous allons exclure, volontairement, de cette phase une dernière étape qui serait celle de l'interprétation. Cependant, nous n'ignorons pas les rapports existants entre les contraintes d'exécution et les contraintes compositionnelles.

\section{I.3 L'esprit et la matière}

Comme nous venons d'exposer, il est possible d'articuler l'acte de composition en deux phases (non disjointes), la phase conceptuelle et la phase d'écriture

Sans doute, ces deux phases ne constitueront pas des mondes cloisonnés, il est évident qu'il existera un grand mouvement entre elles, de façon qu'il y ait une communication et une grande circulation de pensée. Petit à petit l'écriture (au sens matériel, au sens de l'artisanat et du processus) rejoint les concepts et petit à petit les concepts s'adaptent aux contraintes matérielles. Composer sera, effectivement, un mouvement entre ces deux mondes, un échange continue d'informations qui transforment le processus de composition en une opposition permanente entre les concepts et la réalité sonore (ou physique) jusqu'à un état d'équilibre où l'acte se conformera aux concepts et vice et versa. Les concepts et l'écriture convergeront petit à petit à un stade d'équilibre.

L'acte de composition sera une lute (opposition, adéquation...) permanente entre le monde abstrait des concepts et le monde de la réalité sonore, et l'oeuvre finale sera le point de convergence entre ces deux mondes!

La difficulté se présente, alors, dans la transition entre la phase conceptuelle et la phase d'écriture. Comment passer alors de l'idée, du concept à l'écriture, au réel? Les concepts nécessitent d'une intermédiation, d'un biais pour se matérialiser, d'une représentation dans l'univers réel de la musique et des musiciens.

Pour pouvoir comprendre cela, tegardons un peu plus en détail la phase que nous avons appelé la phase d'écriture et essayons de comprendre comment les idées prennent forme.

\subsection{L'écriture}

L'acte de composer (la phase d'ćcriture), au sens traditionnel, pourrait se réduire à une succession de choix "uniques" dus à une intuition instantanée.

Sans doute, ces choix ne sont pas disposés linéairement dans le temps, ni ils sont si neutres comme on pourrait le penser.

Cliaque choix est, certes, préparé par des sélections, d'autres choix, et des explicitations antérieures opérées à plusieurs niveaux, du plus élémentaires au essentiel.

4 Marque Digidesign.

5 BOULEZ, Pierre (1981)- "L'in(dé)fini et l'instant", in Le compositeur es L'ordinateur, IRCAM, Paris. 
Ce moment décisif du choix est dû, alors à celte intuition instantanée ou plutôt à un "sens" trouvé dans une relation entre les objets, (qui à première vue peuvent paraittre disjoints ou discontinus) qui délaisse toutes les autres options de côté.

Alors ce choix, du quel découle un sens, est dû à une multitude de facteurs, entre lesquels:

- Les contraintes préalables, (Le choix doit s'avérer d'être cohérent avec le cadre formel axiomatisé auparavant).

— un sens qui se dégage dans l'acte "d'écrire"

- les relations qui se forment entre les éléments du texte musical au moment des choix, et qui se forment par l'acte

- Le vécu du compositeur.

— Son oreille interne et son esthétique (ou dirais-je ses représentations internes!).

Par conséquent, au début, chaque choix doit préparer les choix suivants, cliaque choix a une mémoire et il est prémonitoire. Mais, au fur at à mesure que la composition avance, I! n'existe plus d'a priori ni d'à posteriori, mais un tissu de relations qui s'auto organisent pour constituer une ocuvre, d'où émerge un sens nouveau, qui échappe à l'axiomatisation "a priori".

Composer scra, au niveau de l'écriture, donner (ou trouver) un sens à (ou dans) un matériau, de façon que l'oeuvre sera un tissu de relations engendrées par ces choix uniques (conscients ou inconscients) faits par le compositeur.

\subsection{Le pont}

Il nous manque, maintenant, un pont, une troisième étape qui puisse permettre la transition. Comment conformer les concepts à la pratique? Quel est le moyen de franchir l'abîme qui sépare les notions abstraites des contraintes du réel? Qu'est-ce qui nous permettra de tisser un lien entre l'univers des abstractions et l'univers des contraintes physiques (materielles et perceptives)?

Pour résoudre ce dilemme nous proposons une troisième phase qui va fédérer le réel et l'abstrait:

\section{La phase de representation ou de modelisarion!}

Cette plase, sera responsable par l'établissement de modk̉les qui permettront au compositeur de représenter concrètement ou musicalement ses concepts abstraits (musicaux ou extrn musicaux). Le modèle (notion que nous allons expliciter dans la suite de ce texte), mème s'il r'śtait pas reconnu comme tel a toujours étí une idée sous-jacente dans l'histoire musicalc, ${ }^{6}$ et souvent lié à ce que nous appelons "l'artisanat compositionnel". La tradition musicale nous en donne un grand nombre d'exemples:

A. Schoenberg:

Concept-> ślimination d'un pôle tonal, trouver un système larmonique qui puisse éviter une prosminence d'une hauteur sur les autres.

Modèle-> L'énumération, sans répétition (au moins au départ), d'une des permutations du total chromatique.

6 MESNAGE M., RIOTTE A. (1993), "Modelisation informatique de partitions, analyse cl composition assistite", in Les Cahiers de I'IRCAM, n' 3 juin 1993, IRCAM, Paris. 
Brian Ferneyhough:

Concept-> l'établissement d'un tissu de relations et de sens entre les divers niveaux de la composition

Modèle $>>$ la combinatoire, la permutation symbolique. L'application d'un méme modèle formel (issu souvent de la combinatoire) sur des ensemble de paramètres musicaux différents. L'utilisation de la combinatoire comme paradigme pour la génération d'un ensemble sémantique maximal avec un matériau minimal.

\section{Boulez:}

Concept-> Un contrepoint issu d'un déphasage entre les paraniètres musicaux. ${ }^{7}$

Modele $>$ construction d'une structure musicale avec un décalage formel dans l'application de permutations sur les divers paramètres musicaux.

\section{Xenakis:}

Concept-> Une conception globale d'une structure sonore

Modèle $>$ L'utilisation du calcul de probabilités pour le calcul des "nuages" sonores.

Murail:

Concept-> La primauté de la perception en vers le formel.

Modèle $>$ L'utilisation de modèles acoustiques en composition.

On peut saisir que le modèle est l'agent fédérateur entre les concepts et la phase d'écriture, entre l'abstrait, l'imaginaire du compositeur et le physique, le sensible, le réel.

\section{Le modèle}

La notion de modele (comme le remarque A. Gire) couvre un champ sémantique complexe, avec un spectre très large de significations, qui va dès des formalismes mathématiques aux maquettes, et aux moules. Mais on perçoit que la nature de médiateur entre les diverses instances du réel apparait comme étant l'un des éléments de l'intersection des divers ensembles símantiques.

Le mot "modèle" vient du latin "modulus", diminutif de "modus". "Modulus" a le sens de "mesure arbitraire', servant à établir des proportions entre les parties d'un ouvrage, des relations quantitatives entre les parties. "Modus" duquel dérive le mot moule, prend un sens de forme, façon et manière (modus faciendi).

Un modèle peut être, alors, plus qu'une représentation abstraite du réel il peut être, aussi, son icône (la maquette) et la façon comme l'abstrait prend forme, la fạ̧on, la "manière de faire" à travers laquelle le compositeur concrétise ses idées.

Avant de continuer il est important de remarquer qu'il n'est pas notre but de dresser un catalogue exhaustif de la sémantique du mot "modèle", mais de lui retrouver le sens d'objet médiateur entre le réel et l'abstrait, et en même temps comme étant un possible médiateur et transcripteur de concepts.

La modélisation fera part intégrante du processus de composition, comme ćtant la phase transitoire entre les concepts et l'écriture, comme étant une part de l'acte d'écriture:

A la base, toute *...composition procède de modeles,... implicites ou explicites..."8

\footnotetext{
7 En ce qui conceme ces "Structures" pour deux pianos.
} 
La modélisation peut, aussi se diviser en deux phases principales:

-La formalisation et l'axiomatisation des concepts qui génèrent un modèle. -L'extrapolation ou le détournement de ce modèle.

Premièrement, le modẻle est construit à partir d'une néflexion theorique et/ou d'une analyse d'autres oeuvres. ${ }^{9}$ Comme par exemple les variations de Paganini revues par Liszt, les symétries appliquées à une série dodécaphonique comme résultat de l'extrapolation que Webem a fait des processus polyphoniques du moyen àge et de renaissance, le déplasage comme idée musicale issue de l'étude de Ligeti des polyphonies rytlmmiques africaines, I'utilisation de modes particuliers chez Bartok à partir de son étude du folklore europén, les modes rythmiques de Messiaen issus de son étude de la rytllmique hindou, etc. Dans la tradition musicale et même dans une grande partie de la production actuelle il est possible de trouver un bon nombre d'exemples de cette sorte: l'analyse, ou l'étude d'une oeuvre et l'extrapolation ou le détournement de ce modèle.

\subsection{Le modèle chez les compositeurs}

A ce moment nous pouvons aflimer que le modele (où l'acte de représenter) fait partie intégrante de l'acte compositionnel". ${ }^{10}$ Que dans ce parcours qui mène le compositeur en vers l'oeuvre (la composition), il passe, forcement, à un moment ou à un autre, par un espace de modèles.

Nous pouvons à guise d'exemples faire un petit parcours chez quelques compositeurs et retrouver cette notion de modèle dans toutes ses couleurs:

- Le modèle formel dans les concertos de Vivaldi.

- Le modẻle onomatopéique dans les oeusres de Jannequin (représentation du réel)

- Le "Mode de valeurs et d'intensités" comme modèle de pensée musicale parannetrée qui a nourri des pièces comme les Structures, Kreuzspiel, Le marteau, etc.. (théorie prescriptive)

- Le concerto en la mineur pour quatre clavecins de J.S. Bach qui est une transcription du Concerto en si mineur pour quatre violons de Vivaldi. (modèle esthétique)

- La manipulation dadécaphonique comme modèle de système compositionnelle pour A. Schoenberg, A. Berg, A. Webern, etc.(théorie prescriptive).

- Le son de la cloche et la voix d'un adolescent comme modele larmonique, de timbre, de forme, et de synthiese dans "Mortuos Piango, Vivo Voco" de J. Harvey.

- Les concepts stochastiques de I. Xenakis matérialisés par le modèle de MaxwellBoltzmann dans Metastasis.

- La série de Fibonnaci comme modèle pour la construction des mesures dans le deuxième quatuor de Brian Ferneythough.

- Le modile sonore qui nourrit bonne partie de l'oeuvre de T. Murail, Grisey (le spectre de trombone dans Partiels)

8 BARRIERE. Jean Baptiste (1989) - "Écriture et modeles" in Entretensps n 8, Paris, France.

9 Pour Boulez composer sera en quelque sorte savoir extrapoler les experiences de nos ainés. Rapjelons-nous aussi des travaux d'analy'se et de formalisation musicales entrepris par A. Riotte et M. Mesnage.

I0 BARRIERE, Jean Baptiste (1990) - "Devenir de l'écriture musicale assistè par ordinateur: furmalismes, formes, aides d la composition", in Revie d"analy'se musicale, n52, “"ine trimestre de 1990. 
-La pièce "Ombres" Pour orchestre à cordes de A. Boucorechliev qui prend son modèle dans les quatuors de Beethoven,

-Un modèle d'écriture, axé sur la polarisation d'une hauteur, qui se dégage dans plusieurs oeuvres de L. Berio, telles que: Sequenza V pour trombone (1966), Sequenza VII pour hautbois (1969), Comma pour clarinette piccolo en mib (1987) et le début de la section XXXI de Coro (1975/1976), duo Baryton et trombone.

\subsection{Les modèles}

De ce que nous venons de voir, musicalement, la notion de modele recouvre un ensemble sémantique assez grand, qui nous ramène à des modèles stylistiques, esthétiques, d'écriture, formels, conceptuels, métaphoriques, etc...

Cependant, comme nous l'avions inféré, la nature de médiateur, fédérateur entre les diverses instances du réel se présente comme un invariant. D'une façon plus pragmatique nous pouvons dire que les modẻles sont:

-des représentations conceptuelles,

- des intermédiaires qui font le lien entre le monde abstrait des idées (concepts) et le monde concret (des sons, des instruments, du cognitif), ils sont les fédírateurs entre l'imaginaire abstrait et le sensible.

-des "manières" de faire.

Le modèle (lui même et non sa notion) peut être le support matériel d'une musique penséc et l'explicitation d'une pratique. Le modèle peut être le regard (où meilleur l'écoute) du compositeur sur la musique, la sienne et celle des autres. L'analyse d'un modèle en particulier permet de pénétrer dans le monde sonore du compositeur, il montre quelles sont les priorités, quels sont les paramètres ou quelles sont les entités qui sont où qui ne sont pas prises en charge, explicitement. Il est, en faite, une entité multi-facétée qui permet l'accès à plusieurs aspects du monde du compositeur:

Son écoute (ou son regard) de la musique, son "modus-faciendi", quels concepts sont-ils explicités, et quels ne le sont pas.

Comme exemple prenons Xenakis. Il utilise des modeles stochastiques, qui sont des modèles globaux, originaires d'une formalisation que Xenakis a fait de son ćcoute de la musique sérielle. Pour cela nous renvoyons le lecteur à l'article "La crise de la musique sérielle" de 1955, publié dans les Gravesanner Blätter n I, où nous pouvons lire:

La polyphonie linśaire se détruit d'elle mêıne par sa complexité actuelle. Ce qu'on entend" n'est en réalité qu'amas de notes à des regisưes variés. La complexité ènorne empèche à l'audition de suivre l'enchevestrement des lignes et a comme effet macroscopique une dispersion irraisonntc et fortuite des sons sur toute l'étendue du spectre sonore. Il y a par conséquent contradiction entre le systéme poly* phonique linéaire et le résultat entendu qui est surface, masse.

Il est surprenant de lire ce passage puisqu'il nous donne l'impression que Xenakis parle de sa propre musique. Finalement les deux qualifications qu'il utilise pour qualifier "Ce qu'on entend", surface et masse, sont exactement les deux concepts qui lui seront les plus chers, il nous

"I If faudrait lire ici: "Ce que j"entend..". 
suflit de penser à Metastasis, Phytoprakta, etc.. En outre les deux concepts utilisés sont des représentations géométriques, en deux et trois dimensions, issues de son expérience d'architecte.

Un autre aspect qui pourra tenir notre attention: Le modèle d'un compositeur gènúralement filtre les autres modêles, ce qui occasionne des analyses d'un compositeur en vers un autre de ce genre:

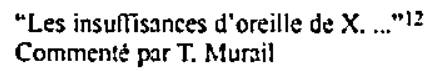

Dans ce cas précis l'intersection des modèles utilisés par Tristan Murail et Iannis Xenakis est, aux niveaux des hauteurs, presque nulle!

L'écoute de Tristan Murail est une écoute fréquentielle tris raflinée, pendant que l'écoute de lannis Xenakis est une écoute globale qui s'intéresse plutôt aux aspects de texture, surlace et masse. Cette considération peut nous montrer que ni Jannis Xenakis est sourd, ni Tristan Murail n'a rien compris du travail de lannis Xenakis, le fait qui s'impose est que les priorités de l'un en de l'autre son divergentes, les formes de représentation avec lesquelles ils perçoivent le monde musical ne sont pas convergentes.

Toutes ces digressions ne doivent pas nous conduire à penser qu'il existe un parcours direct: Concepts $>>$ modeles $>>$ composition... Ce que nous voulons dire est que dans le trajet que le compositeur fait pour aller de son monde conceptuel, abstrait pour arriver à la composition, il devra passer par un espace que nous avons appelé "l'espace de modélisation".

\subsection{Un espace de modèles}

En fonction des exemples donnés, 11 est possible, maintenant, de définir un espace de modélisation, ${ }^{13}$ en définissant quatre grandes catégories de modẻles:

-Les modèles logiques: Ce sont les modeles qui tiennent de relations logiques at abstraites, par exemple:

Les modèles combinatoires de la musique sérielle.

Les modèles probabilistes de la musique stochastique.

-Les modèles analogiques: ce sont les modèles qui ont leur origine dans des modèles pour d'autres entités du réel, par exemple:

Les modelles fréquentielles de la musique spectrale qui tiennent de l'acoustique,

Les modèles de la théorie des gaz utilisés par Xenakis dans Metastasis

-Les modẻles métaphoriques: ce sont les modèles qui opèrent par analogies, souvent du langage, par exemple:

Les programmes littéraires ou poétiques appliqués à certaines compositions.

-Les modèles phoriques: ce sont les non-représentations, ils replissent une partic de l'espace de modélisation où la représentation rejoint le représenté, où le concept rejoint le rél.

\footnotetext{
12 MURAIL T. (1989) - "Questions de Cible" in Emrelemps $1^{\circ}$ 8, Paris, France.

13 GIRÉ Alain (1987) - Modeles mathématiques de șistèmes évolutifs héréditair's, Presses Universitaires de Lyon, p. 271-273.
} 
c'est-à-dire c'est l'objet qui se porte à lui même! Comme exemple nous avons l'attitude de Boucorechliev (dans son hommage à Beethoven) avec sa pièce OMBRES, l'attitude de Stravinsky dans ses visites à Pergolesi ou, finalement Les concertos-pastiches du jeune Mozart. Dans tous ces cas le son, une esthétique ou une musique a été le modèle. Nous pouvons inclure les modèles stylistiques et esthétiques.

Ces quatre catégories finissent par créer un espace de modélisation que le compositeur parcours souvent dans sont processus de création, soit en formalisant (représentant) ses concepts à travers des différents modèles, soit en dialoguant avec ces mèmes modeles de façon à créer un mouvement d'information qui permet que ces représentations proposent des nouveaux concepts.

\section{L'IMPORTANCE DE CETTE RÉFLEXION}

Dans notre travail seulement une partie de cet espace retiendra notre attention, ça sera la partie qui se réfere aux modèles logiques et analogiques. Cettes catégories de modéles, en défaut des autres, nous permettent d'avoir des modèles calculables, ce qui est un fait non négligeable, comme nous verrons, dans l'utilisation de la CAO (la composition assistée par ordinateur).

\subsection{Le compositeur et l'ordinateur}

Un des problèmes principaux en C.A.O. concerne la diffërence existante entre le langage que se sert l'homme pour s'exprimer, 14 soit le langage naturel, oral et écrit, imprégné souvent d'analogies, de métaphores et d'ambiguittés et les langages formels qui rëgissent le comportement des ordinateurs.

Le compositeur évolue normalement dans le royaume du sens, des significations, où la syntaxe est au service de la sémantique. La langue qu'il utilise pour s'exprimer possède une grammaire, un lexique raisonnablement stable, des règles de syntaxe. Cependant, ce qui est plus important, cette langue possède (ou permet) une grande marge d'ambiguĩté. Ambiguïté, qui permet des analogies, des métaphores et toutes autres formes de relations incertaines, desquelles se nourri, souvent, l'imaginaire du compositeur. Is II ne faut pas oublier que l'ambiguïté, l'inespéré ct l'irrégularité font partie de l'oeuvre d'art.

Par contre l'ordinateur appartient à un autre monde, un monde purement syntaxique ${ }^{16}$ régi par des règles strictes de transformation et de calcul. Cet univers, très particulier, ne tolère pas l'ambiguïté. Tout message envoyé à un système quelconque doit être conforme à un code préétabli. Les instructions doivent être formellement explicitées sans ambiguïté, car la machine ne sait pas interpréter des ordres en fonction de situations particulières ou de contextes généraux (sauf, bien sûr si cela a été prévu dans sa programmation!).

Pour beaucoup de compositeurs, la formalisation pouvait en être qu'un choix, mais avec la C.A.O., elle en est devenue une nécessité, un outil, un besoin impératif pour communiquer avec la machine.

I4 LEVY, Pierre (1987)-La Machine Univers, Editions la Dicouverte, Paris-France.

is BOULEZ P., GREUSSAY P. (1987), "ET LA MUSIQUE", entretient avec Philippe MANOURY in TRAI'ERSES +4-45, Machines lirtuelles, Centre de Création Industrielle, Cenure Georges Pompidou, Paris.

16 N'oublions pas que nous sommes en train de parler d'ordinateurs avec ce qu'on appelle une architecture de Von-Neuman déterministe, ce qui exclue toute autre architecture telle que celle de réseaux néturonaux, des très récents ordinateurs moléculaires et toute autre architecture qui puisse gérer des informations "floues". Cette exclusion, volontaire, est dû a la seule raison que la grande majorité, sinon la total itê des machines disponibles sur le marche, à ce moment (1995/1996) ne possède qu'une architecture dite de Von-Neuman. 
Cette réflexion nous amene à nous rendre compte des diffërences existantes entre les

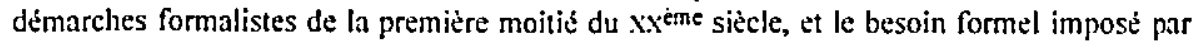
l'ordinateur en C.A.O.

Les unes étaient fondées sur la thèse, implicite, d'une isomorphie entre le monde des relations musicales et le monde des relations formels, intluence poussée de la pensée dominante dans le monde dit "scientifique" et d'une attitude classifice de "neo-pythagoricienne" par certains compositeurs tels que Michel Philipot. ${ }^{17}$ Cette conception réductionniste, où toute tentative pour connaitre quelque chose de l'homme doit d'abords passer par une réduction de l'expérience à un système de marques corrélatives, ${ }^{18}$ à amenée certaines musiques à une illusion formaliste, qui finissait par conférer aux modèles et aux symboles un privilège ontologique sur les processus qu'ils représentaient.

Nous ne voulons pas affirmer ici que cette façon d'agir exclue l'existence d'une conceptualisation à un niveau musical. Dans beaucoup de cas, la paramétrisation de l'espace sonore était, aussi une manière, de concrétiser des idées musicales. Pour Boulez, par exemple, le mécanisme sériel a servi comme outil formel pour concrétiser le concept de contrepoint structurel, oủ la structure sonore était engendrée par une indépendance, contrölée, des diverses dimensions musicales. ${ }^{19}$ D'un autre côté, Xenakis amène au bout l'idée d'indépendance des paramètres musicaux en appliquant des modèles statistiques pour formaliser le concept de "nuages". De notre côté nous avons déjà montré la possibilité d'utiliser des modèles mathématiques en tenant compte des ses caractéristiques, de manière à pouvoir les utiliser, non pas comme des simples algorithmes, ${ }^{20}$ mais comme des outils de formalisation de concepts musicaux. ${ }^{21}$

La grande nouveauté dans l'utilisation de l'ordinateur en musique, dans cette deuxième moitié du $x^{\text {crme }}$ siècle, ne consiste pas dans la formalisation de la théorie, mais dans la formalisation de la pratique, du métier, le royaume traditionnel de l'expérience personnelle, de l'intransmissible.

Actuellement le métier compositionnel hérite, pour ainsi dire, de quelques unes de ces pratiques, mais se rend compte que, spécialement dans le cas de la C.A.O., la formalisation (la modélisation) n'est plus seulement un support intellectuel à la musique, mais devient un outil, indispensable, pour diminuer les distances dans la communication homme-machine.

17 PIILIPPOT M. (1963) - "La certitude et la Foi", in Jannis .Yenakis at la Mhusique Stochastique, La Revie 1insicale- Camet critique 257, Paris.

18 GRANGER, Giles Gaston (1958) - La Pensée formelle et les sciences de l'homme, Aubier-Montaigne.

19 Nous pourrions comme mène nous poser la question de savoir si la combinatoire était, ou est le meilleur modele pour matérialiser ce concept.

20 BIDLACK, Rick (1992). "Chnotic Systems as Simple (but complex) Compositional Alguritlsms", in Computer Afusic Journal, vol 16, $n^{\circ} 3$, Fall 1992.

21 MALT. M. (1994), "Modëlos Matemáticos e Composição Assistida por Computador, Sistemas Estocásticos e Sistemas Caóticos", in Primeiro Simpósio de Computçcio e Mirsica, Caxambu, MG. Brésil.

MALT, M. (1995), "Descente au Paradis Notes sur la réalisation musicala de la bande pour la séquence vidéo Descente au Paradis de Sabine PORADA", in Detxiemes Journees difformatique Mlusicale JIMI "95, Jussieu, Paris.

M1ALT, M. (1996), "Lambda3.99 (Chaos et Composition Musicale)", in Troisiëmes Journes d"lnformatique" Musicale $J I M \cdot 96$. lle de Tatilow, Normandie, France. 